\title{
Current perspectives on the mechanisms of auditory hallucinations: introduction to the special research topic
}

\author{
Johanna C. Badcock ${ }^{1 *}$, Frank Larøi ${ }^{2}$, Kelly Diederen ${ }^{3}$ and Paul Allen ${ }^{4}$ \\ 1 School of Psychology, University of Western Australia, Crawley, WA, Australia \\ 2 Department of Psychology, Cognition and Behaviour, University of Liège, Liège, Belgium \\ ${ }^{3}$ Department of Physiology, Development and Neuroscience, University of Cambridge, Cambridge, UK \\ ${ }^{4}$ Institute of Psychiatry, King's College, London, UK \\ *Correspondence: Johanna.badcock@uwa.edu.au
}

Edited by:

Hauke R. Heekeren, Freie Universität Berlin, Germany

Keywords: auditory hallucinations, cognition, emotion, hearing voices, non-clinical hallucinations, psychosis, schizophrenia

According to the Oxford English Dictionary (http://www.oed. com) the term "perspective" is derived from the verb perspicere (from per- "through" + specere "to look"), meaning "looked at closely." In keeping with this origin, the contributors to this Frontiers Research Topic have indeed looked closely at the experience of auditory hallucinations $(\mathrm{AH})$, providing new insights into the precise nature of hallucinations in clinical and non-clinical groups; the underlying cognitive, emotional and neural processes; and how this evidence might inform the next generation of personalized treatments Connor and Birchwood (2013).

The phenomenology of $\mathrm{AH}$ and the mechanisms that underpin them lie at the heart of our research, yet there has been a growing concern that the characteristics of $\mathrm{AH}$ prioritized by researchers don't match those considered important to hallucinators themselves. To some degree this disparity reflects the (sometimes) limited scope of popular research assessment tools. However, disregarding the phenomenological diversity of $\mathrm{AH}$ may guarantee that we will fail to understand the biopsychosocial processes involved. Consequently, the resurgence of interest in phenomenological research reflected in current contributions is both timely and welcome. McCarthy-Jones et al. (2013) call for more nuanced descriptions of AH that reflect more accurately what the experience is like, and proffer a philosophical phenomenological perspective, which encourages a systematic exploration of (normal and $\mathrm{AH}$ ) experiences from the first person perspective. Both Badcock and Chhabra (2013) and deLeedeSmith and Barkus (2013) tackle the diverse presentations of AH in clinical and non-clinical groups. deLeede-Smith and Barkus adopt a developmental perspective, charting how the features of AH emerge and persist across the lifespan, and suggest that mechanisms maintaining $\mathrm{AH}$ differ across these populations, whilst Badcock and Chhabra (2013) provide an extensive and reflective review of the literature on the perception of voice identity, which points to subtle biases across different levels of voice identity. Rounding out this set of articles, Stephane (2013) emphasizes the heterogeneity of $\mathrm{AH}$ from one voice hearer to the next, and persuasively argues that whilst hallucinatory experiences are unique to each individual they are not random collections of features. Instead, Stephane suggests, they arise from a limited number of dimensions of phenomenological AH space-each linked to a separate neural basis.
Continuing the neural processing theme, Kompus et al. (2013) show that while non-clinical voice hearers present with a reduced response to speech sounds in the primary auditory cortex, attentional modulation of this area is intact. In strong contrast, no attentional modulation of this area could be observed in schizophrenia patients with AH. Further similarities as well as differences in mechanisms underlying $\mathrm{AH}$ are shown by Dahoun et al. (2013) who compared neural correlates of mentally simulated actions (e.g., "open a window") between hallucinationprone adolescents and a group with a genetic risk for schizophrenia (22q11.2 deletion syndrome). While both groups exhibited decreased activation in regions related to self-other distinction when imagining a close friend performing an action, individuals with a genetic risk for schizophrenia displayed additional decreased activations in areas associated with visual imagery, episodic memory and social cognition when "simply" seeing a cue that said either "you" or "best friend" earlier on in the task.

Another set of articles highlight the role of emotion in AH. Kanemoto et al. (2013) show that university students with high hallucination proneness tended to make more external misattributions of inner thoughts than those with lower hallucinations proneness. Importantly, they also show that emotional valence affected the ability to recall whether a word had been previously heard, or had been imagined, only in the latter group of subjects. Similarly, with the help of an implicit emotional prosody task, Alba-Ferrara et al. (2013) examine how attention is involuntarily captured in patients with schizophrenia and healthy controls, and conclude that patients with $\mathrm{AH}$ may be less lateralized in their processing of emotion conveyed in voice. Finally, based on studies that have demonstrated that patients with schizophrenia have difficulty perceiving and discriminating emotions based on affective prosody compared to healthy controls, Tucker et al. (2013) wished to examine whether this may also represent an endophenotype of schizophrenia, i.e., by examining this capacity in non-affected, first-degree relatives. They found that unaffected relatives of AH schizophrenia patients, compared to the matched healthy controls, exhibited some basic impairments in auditory processing, suggesting that auditory processing deficits may be a core feature of $\mathrm{AH}$ in schizophrenia, but that the two groups did not differ significantly on a major variable, i.e., number of errors in pitch discrimination. Clearly, more research is needed in 
order to examine whether these difficulties represent a potential endophenotype for $\mathrm{AH}$.

Finally, from a neurofunctional perspective, two papers report new findings in patients with schizophrenia and auditory verbal hallucinations (AVH). Homan et al. (2013) used Arterial Spin labeling (ASL) - a Magnetic Resonance technique for measuring cerebral blood flow (CBF) - to show that increased perfusion in the left superior temporal gyrus (STG; a cerebral area known to support language and auditory function) in patients with schizophrenia and $\mathrm{AVH}$, relative to controls and global $\mathrm{CBF}$ in patients, was not reduced by treatment with transcranial magnetic stimulation even though AVH symptom scores decreased over the treatment period. These findings are consistent with what has previously been termed a trait marker of AVH in schizophrenia. The second paper by Koeda et al. (2013) used a novel functional MRI favorability judgment task to show that hypo-activation in the left STG may be associated with brain dysfunction in accessing vocal attractiveness in schizophrenia, although right fronto- parietal regions could offset STG dysfunction associated with social communication.

In sum, the variety of perspectives featured in this Research Topic illustrates the vitality of current research on $\mathrm{AH}$ across a range of diagnostic groups, and the significant advances that have been made in understanding the mechanisms the underlie them.

\section{REFERENCES}

Alba-Ferrara, L., de Erausquin,G. A., Hirnstein, M., Weis, S., and Hausmann, M. (2013). Emotional prosody modulates attention in schizophrenia patients with hallucinations. Front. Hum. Neurosci. 7:59. doi: 10.3389/fnhum.2013. 00059

Badcock, J. C., and Chhabra, S. (2013). Voices to reckon with: perceptions of voice identity in clinical and non-clinical voice hearers. Front. Hum. Neurosci. 7:114. doi: 10.3389/fnhum.2013.00114

Connor, C., and Birchwood, M. (2013). Through the looking glass: self-reassuring meta-cognitive capacity and its relationship with the thematic content of voices. Front. Hum. Neurosci. 7:213. doi: 10.3389/fnhum.2013.00213

Dahoun, T., Eliez, S., Chen, F., Badoud, D., Schneider, M., Larøi, F., et al. (2013). Action simulation in hallucination-prone adolescents. Front. Hum. Neurosci. 7:329. doi: 10.3389/fnhum.2013.00329
deLeede-Smith, S., and Barkus, E. (2013). A comprehensive review of auditory verbal hallucinations: lifetime prevalence, correlates and mechanisms in healthy and clinical individuals. Front. Hum. Neurosci. 7:367. doi: 10.3389/fnhum.2013.00367

Homan, P., Kindler, J., Hauf, M., Walther, S., Hubl, D., and Dierks, T. (2013). Repeated measurements of cerebral blood flow in the left superior temporal gyrus reveal tonic hyperactivity in patients with auditory verbal hallucinations: a possible trait marker. Front. Hum. Neurosci. 7:304. doi: 10.3389/fnhum.2013.00304

Kanemoto, M., Asai, T., Sugimori, E., and Tanno, Y. (2013). External misattribution of internal thoughts and proneness to auditory hallucinations: the effect of emotional valence in the Deese-Roediger-McDermott paradigm. Front. Hum. Neurosci. 7:351. doi: 10.3389/fnhum.2013.00351

Koeda, M., Takahashi, H., Matsuura, M., Asai, K., and Okubo, Y. (2013). Cerebral responses to vocal attractiveness and auditory hallucinations in schizophrenia: a functional MRI study. Front. Hum. Neurosci. 7:221. doi: 10.3389/fnhum.2013.00221

Kompus, K., Falkenberg, L. E., Bless, J. J., Johnsen, E., Kroken, R. A., Kråkvik, B., et al. (2013). The role of the primary auditory cortex in the neural mechanism of auditory verbal hallucinations. Front. Hum. Neurosci. 7:144. doi: 10.3389/fnhum.2013.00144

McCarthy-Jones, S., Krueger, J., Larøi, F., Broome, M., and Fernyhough, C. (2013). Stop, look, listen: the need for philosophical phenomenological perspectives on auditory verbal hallucinations. Front. Hum. Neurosci. 7:127. doi: 10.3389/fnhum.2013.00127

Stephane, M. (2013). Auditory verbal hallucinations result from combinatoric associations of multiple neural events. Front. Hum. Neurosci. 7:239. doi: 10.3389/fnhum.2013.00239

Tucker, R., Farhall, J., Thomas, N., Groot, C., and Rossell, S. L. (2013). An examination of auditory processing and affective prosody in relatives of patients with auditory hallucinations. Front. Hum. Neurosci. 7:531. doi: 10.3389/fnhum.2013.00531

Received: 12 October 2013; accepted: 30 October 2013; published online: 18 November 2013.

Citation: Badcock JC, Larøi F, Diederen K and Allen P (2013) Current perspectives on the mechanisms of auditory hallucinations: introduction to the special research topic. Front. Hum. Neurosci. 7:792. doi: 10.3389/fnhum.2013.00792

This article was submitted to the journal Frontiers in Human Neuroscience.

Copyright (c) 2013 Badcock, Larøi, Diederen and Allen. This is an open-access article distributed under the terms of the Creative Commons Attribution License (CC BY). The use, distribution or reproduction in other forums is permitted, provided the original author(s) or licensor are credited and that the original publication in this journal is cited, in accordance with accepted academic practice. No use, distribution or reproduction is permitted which does not comply with these terms. 